\title{
The history of polarisation measurements: their role in studies of magnetic fields
}

\author{
R. Wielebinski \\ Max-Planck-Institut für Radioastronomie, Auf dem Hügel 69, 53121 Bonn, Germany
}

Radio astronomy gave us new methods to study magnetic fields. Synchrotron radiation, the main cause of comic radio waves, is highly linearly polarised with the ' $\mathrm{E}$ ' vector normal to the magnetic field. The Faraday Effect rotates the ' $\mathrm{E}$ ' vector in thermal regions by the magnetic field in the line of sight. Also the radio Zeeman Effect has been observed.

At first radio sources were shown to have a non-thermal spectrum. Solar observers detected polarisation in earliest observations. The 'Rosetta stone' of the magnetic fields was the Crab Nebula. The prediction that the Crab Nebula was a synchrotron source was confirmed first in 1954 by optical polarisation observations and in 1957 at radio frequencies. The year 1962 was a great year for magnetic field research: the polarisation of the Galaxy was detected as well as the polarisation of radio galaxies. In 1968 pulsars were discovered, highly polarised sources. Both pulsars and compact radio sources can be used in conjunction with the Faraday Effect to probe interstellar medium. Also in 1968 the detection of the first radio Zeeman Effect was announced.

In the 1970s several new large radio telescopes became operational. All of these telescopes were capable of polarisation observations. The Westerbork radio telescope first detected polarisation in the galaxy M51. The Effelsberg 100-m dish was ideal for studying the diffuse polarised emission of the Galaxy, galaxies and radio galaxies. The Ryle telescope and the VLA made polarisation maps radio galaxies. For some time only the Parkes radio telescope was available for polarisation studies of southern sources. The Australia Telescope came later with good polarisation mapping facilities.

Polarised sources were used to delineate magnetic fields of the Galaxy. Pulsars were favoured for such studies. More recently new data became available from synthesis instruments for extragalactic sources along the Galactic plane to allow more detailed studies. Also the increase of the numbers of Rotation Measures at high Galactic latitudes allow studies of the halo magnetic field.

All-sky surveys in polarisation were made by the WMAP satellite at high radio frequencies (from $23 \mathrm{GHz}$ to $94 \mathrm{GHz}$ ) with medium angular resolution. Also a $1.4 \mathrm{GHz}$ all-sky polarisation survey was made. High resolution low frequency polarisation observations showed rapid variation of the ' $\mathrm{E}$ ' vector implying high rotation measures leading to complete depolarisation. Zeeman Effect observations have been extended to various molecular species, some at mm-wavelengths. The advent of new digital devices led to the development of the Rotation Measure synthesis. This development allows the search in depth of interstellar magnetic field features.

The future of magnetic field research is in good hands. All the important new 'megatelescopes' have magnetism as key science. The first E-VLA polarization map of NGC 4631 has been published. The LOFAR and ALMA radio telescopes have polarisation capabilities. The advent of the two Square Kilometre Array pathfinders ASCAP and MeerKAT, followed by the final SKA array one day will enhance greatly the studies of cosmic magnetic fields. 